\title{
L'EXPLOITATION MINIERE, LA DECENTRALISATION ET LE DEVELOPPEMENT DE LA VILLE DE KOLWEZI
}

\author{
Par Vital Pius KAJAMA.
}

\section{INTRODUCTION}

La résolution 1803 de l'assemblé générale de Nations- unies du 14 décembre 1962, la charte des Droits et devoirs économiques des États, le pacte International relatif aux droits économiques, sociaux et culturels, le pacte international relatif aux droits sociaux et politiques ainsi que la charte Africaine des Droits de l'homme et des peuples en vigueur le 21 octobre 1986, reconnaissent au niveau international le droit de souveraineté permanente des peuples et des Nations sur leurs richesses et leurs ressources naturelles, lequel droit doit s'exercer dans l'intérêt du développement national et du bien-être de la population de chaque État intéressé sans connaître la moindre restriction pour un quelconque motif.

Ainsi, la violation des droits souverains des peuples et des nations sur leurs richesses et leurs ressources naturelles, va à l'encontre des principes de la charte de Nations-Unies et reste poursuivable devant la commission des Droits de l'homme instituée par l'article 28 du Pacte international relatif aux droits civils et politiques des peuples.

Étant donné que, toute exploitation minière fait l'objet des retombées à la fois positives que négatives au sein du milieu environnemental de manière multisectorielle, tous les chantiers indispensables doivent l'être en vertu de l'ultime but à savoir, celui de l'amélioration des conditions socio-économiques du citoyen, entendons par-là le fils du territoire.

La constitution du 18 février 2006 consacre « la libre administration des provinces et des entités territoriales décentralisées, de leurs compétences et de leurs ressources » (article 123, point 1). Ces entités «sont dotées de la personnalité juridique et sont gérées par les organes locaux » (article 3, alinéa $1^{\mathrm{er}}$ ). Entités parmi lesquelles figure la ville de Kolwezi.

La constitution actuelle de la RDC pose le principe d'une large décentralisation. Cependant, le débat suscité par l'interprétation de l'article 175, en ce qui concerne la retenue à la source par les provinces des 40 pour cent des recettes à caractère national relance la cacophonie toujours vive dans ce pays quant à la perception de la décentralisation, à sa portée exacte (théorique et pratique) et à son incidence sur le développement des entités locales. D'une façon générale, l'on se pose la question de savoir pourquoi la décentralisation territoriale? En RDC, on se demande pourquoi y revenir encore et toujours? Le fait de la prévoir dans la constitution peut-il contribuer à la rendre un outil de développement à la base? 
En effet, lorsqu'on jette un coup d'œil rétrospectif sur l'organisation politico-administrative et la gestion des entités territoriales dans ce pays, ${ }^{1} 1$ 'on se rend compte que, malgré l'échec de sa pratique, la décentralisation territoriale demeure une nécessité impérieuse pour ce pays-continent (2 $345410 \mathrm{~km}^{2}$ ). Depuis les années 1980, la décentralisation comme politique publique n'a pas encore réussi à se constituer en outil privilégié pour le développement des entités locales. Pourtant, les aspirations populaires au partage (solidarité nationale) et à l'élargissement du cercle politique (démocratie, libres initiatives) n'ont pas encore trouvé un impact réel au niveau de l'arrière-pays. Dans ce contexte, on peut se demander comment peut-on développer les entités locales d'un pays si vaste dépourvu des capitaux productifs comme la RDC?

La réponse est simple et renvoie à l'idée selon laquelle l'Etat a un rôle capital à jouer dans tout processus de développement national par le biais, en l'occurrence, de l'administration (territoriale), son épine dorsale.

En effet, de tous les maillons du pouvoir, la territoriale semble en constituer une sorte de centre, au regard de son incidence directe sur le vécu quotidien de la population et des effets induits possibles de son action sur les autres secteurs de la vie sociale. La décentralisation constitue, pour ainsi dire, le mode de gestion le mieux indiqué pour les entités locales au regard des objectifs de reconstruction nationale. Car dans son apprentissage, la décentralisation revêt des aspects pratiques (politique, économique, juridique ou administratif et managérial) qui renferment une certaine dynamique de changement capable de transformer la société tout entière. Pour leur réalisation, les objectifs requièrent la présence des acteurs opérationnels, tant au niveau central que local, ayant « la culture de décentralisation » et pouvant agir en véritables agents de développement.

Cependant, malgré le partage des compétences établi par les textes légaux dans le domaine du développement, ${ }^{2}$ la pratique sociale en matière de décentralisation en RDC a montré que les entités locales y sont aux prises avec l'insuffisance des recettes entraînant ainsi des exactions et la malversation. ${ }^{3}$

Ce qui a empêché, empêche et empêchera encore pendant longtemps les entités décentralisées congolaises à prendre un élan nouveau pour leur auto-développement. Dès lors, l'administration locale congolaise, étouffée dans son rôle d'initiateur des projets autofinancés et d'investisseur public, ne peut se contenter que de son rôle d'animateur et d'encadreur, rôle qu'elle peut jouer correctement. La mobilisation des masses laborieuses ou l'in-

1 Léon De Saint Moulin, «Histoire de l'organisation administrative du Zaïre », in Zaïre-Afrique, $\mathrm{n}^{\circ}$ 224, avril 1988, pp.197-221.

2 La loi no 82/006 du 25 février 1982. voir Epee Gambwa et Otemikongo Mandefu, art. cit., pp. 347-356 ou Norbert Yamba-Yamba Shuku, Les dynamiques de la décentralisation en RDC, Kinshasa, inédit, 2005:5-18.

3 Ekili Tabu et OTemikongo Mandefu, « La décentralisation administrative et les finances publiques zaïroises (cas de la région du Haut-Zaïre », in Zaïre-Afrique, n² 242, février 1990:69-70, 72-74, 76-80;. 
vestissement-travail s'avère une nécessité impérieuse pour enrayer la pauvreté qui sévit dans les entités locales congolaises.

Pour étayer ce point de vue, il serait intéressant de clarifier le contenu des conceptsclefs dont nous nous servons en les contextualisant. Nous allons ainsi situer la décentralisation par rapport à ses aspects pratiques, concrets, avant de dire un mot sur le développement endogène des entités locales. Dans un deuxième temps, nous allons tenter d'appréhender les rapports qui existent ou peuvent exister entre la décentralisation et le développement endogène. Un troisième et dernier volet va saisir le rôle de la mobilisation des masses laborieuses dans le développement des entités locales congolaises. Une brève conclusion va suspendre cette étude.

\section{GENERALITES}

\subsection{Les phases d'un projet minier.}

Du début des prospections minérales à la période de l'après-clôture de la mine, on distingue différentes phases dans un projet minier. Ce qui suit représente les phases typiques d'un projet minier. Chaque phase d'exploitation minière est associée à différents groupes d'impacts environnementaux.

\subsubsection{Prospection.}

Un projet minier peut commencer seulement quand on connaît l'extension et la valeur du dépôt de minerai. Les informations sur la localisation et la valeur du dépôt de minerai s'obtiennent durant la phase de prospection Cette phase comprend les enquêtes, les études de terrain, les essais de sondage et d'autres excavations exploratoires.

La phase de prospection peut entraîner le nettoyage de vastes aires de végétation (typiquement en lignes) pour faciliter la circulation de véhicules lourds transportant les installations de forages.

Plusieurs pays requièrent une Étude d'Impacts Environnementaux (EIE) séparée dès la phase exploratoire d'un projet minier parce que les impacts de cette phase peuvent être profonds et parce que les prochaines phases du projet minier peuvent ne pas s'ensuivre si l'exploration n'arrive pas à trouver des quantités suffisantes de dépôts de minerai à hautes teneurs.

\subsubsection{Développement.}

Si la phase d'exploration prouve l'existence d'un dépôt de minerai assez important et d'une teneur suffisante, le promoteur de projet peut alors commencer de planifier le développement d'une mine. Cette phase du projet minier comprend plusieurs composantes distinctes. 


\subsubsection{Construction de routes d'accès.}

La construction de routes d'accès, soit pour amener les équipements lourds et les approvisionnements au site minier ou bien pour expédier les projets miniers proposés varient en fonction des types de métaux ou de matériaux à extraire de la terre. La majorité des projets miniers proposés concerne l'extraction de minerais tels que: Cuivre, nickel, Cobalt, Or, Argent, Plomb, Zinc, Molybdène et Platine. Ce Guide traite des impacts environnementaux des grands projets miniers relatifs à ces minerais. Ce Guide ne discute pas l'exploitation des minerais qui sont souvent extraits en utilisant les méthodes d'exploitation minière par décapage direct des couches incluant l'aluminium (bauxite), le phosphate et l'uranium.

Les métaux et minerais traités, peut engendrer des impacts environnementaux substantiels spécialement si les routes d'accès sont construites à travers des zones écologiquement sensibles ou près de communautés précédemment isolées. Si une proposition de projet minier comprend la construction de routes d'accès quelconque, alors il faut inclure dans l'EIE du projet une évaluation complète des impacts environnementaux et sociaux de ces routes.

\subsubsection{Préparation et déblaiement du site.}

Si le site d'une mine se situe dans une zone sous-développée et difficile d'accès, le promoteur du projet peut avoir besoin de commencer le déblaiement des terrains pour la construction des zones de campement pour héberger le personnel et stocker les équipements. Même avant de procéder à une quelconque opération de minage de terrains, les activités associées à la préparation et au déblaiement du site peuvent avoir des impacts environnementaux significatifs, surtout si elles se trouvent dans le voisinage des zones écologiquement sensibles. L'EIE doit évaluer séparément les impacts associés à la préparation et au déblaiement du site.

\subsubsection{Exploitation minière active}

Dès qu'une compagnie minière a construit des routes d'accès et préparé les zones de campement pour héberger le personnel et stocker les équipements, les travaux miniers peuvent commencer. Tous les types de travaux miniers partagent un aspect commun: l'extraction et la concentration (Ou enrichissement) d'un métal en provenance du sol. Les projets miniers proposés diffèrent considérablement par les méthodes proposées pour l'extraction et la concentration du minerai métallique.

Dans presque chaque cas, les minerais métalliques sont emprisonnés sous une couche de sol ou de roche ordinaire (appelée 'morts terrains' ou 'débris de roche') qui doivent être déplacés ou excavés pour permettre l'accès au dépôt de minerai.

La première façon dont les projets miniers proposés diffèrent entre eux est la méthode proposée pour déplacer ou excaver les morts terrains. Les paragraphes qui suivent discutent brièvement des méthodes les plus communes. 


\subsubsection{Exploitation à ciel ouvert}

L'exploitation à ciel ouvert est un type d'exploitation dans laquelle le dépôt de minerai s'étend profondément dans le sous-sol nécessitant l'enlèvement de couches superposées de morts terrains et de minerai.

Dans plusieurs cas, l'exploitation des arbres, la coupe rase ou le brûlage de la végétation surplombant le dépôt de minerai peuvent précéder l'enlèvement des morts terrains. L'utilisation de la machinerie lourde généralement des bouteurs/bulldozers et camionsbennes, est le moyen le plus commun pour enlever les morts terrains.

L'exploitation à ciel ouvert fait souvent intervenir le déplacement de la végétation native des zones, et se trouve donc parmi les types d'exploitation minière les plus destructives du point de vue environnemental, spécialement dans les forêts tropicales.

Étant donné que la méthode d'exploitation à ciel ouvert est utilisée pour des minerais se trouvant à une profondeur substantielle, elle fait couramment intervenir la création d'un puits qui s'étend en dessous du niveau de la nappe phréatique. Dans ce cas, l'eau de fond doit être pompée pour permettre les travaux miniers. D'ordinaire, un lac de puits de mine se forme à un certain moment de la durée après la fermeture de la mine et l'arrêt du pompage de l'eau de fond.

\subsubsection{Exploitation des Places}

L'exploitation des places est utilisée lorsque le métal d'intérêt est associé aux sédiments dans un lit de cours d'eau ou dans une plaine d'inondation. Des bouteurs, des dragues ou des jets hydrauliques (un processus appelé 'abattage hydraulique') sont utilisés pour extraire le minerai. L'exploitation des placers vise généralement à récupérer de l'or à partir des sédiments de cours d'eau et des plaines inondables. Parce que l'exploitation des placers se produit souvent dans un cours d'eau, c'est un type d'exploitation minière destructif pour l'environnement, parce qu'il libère de grandes quantités de sédiments qui peuvent avoir des impacts sur les eaux de surface à plusieurs kilomètres en aval de la mine de placer.

\subsubsection{Exploitation souterraine}

Dans l'exploitation souterraine, une quantité minimale de morts terrains est enlevée pour accéder au dépôt de minerai. L'accès à ce gisement de minerai se fait au moyen de tunnels ou de puits. Tunnels ou puits conduisent à un réseau plus horizontal de tunnels souterrains qui accèdent directement au minerai.

Dans une méthode d'exploitation souterraine dénommée 'exploitation par paliers' ou 'méthode d'exploitation par blocs foudroyés', des sections ou des blocs de roche sont supprimés en bandes verticales, ce qui laisse une cavité souterraine connectée qui est généralement remplie avec des agrégats cimentés et déchets de roche. Bien que l'exploitation minière souterraine soit une méthode moins destructive de l'environnement pour accéder à un gisement de minerai, elle est souvent plus coûteuse et comporte des risques de sécurité plus 
élevés que l'exploitation à découvert par décapage direct, y compris l'exploitation à ciel ouvert. Bien que la plupart des projets miniers à grande échelle impliquent l'exploitation à ciel ouvert, de nombreuses grandes mines souterraines sont en opération dans le monde entier.

\subsubsection{Réouverture des mines inactives ou abandonnées et retraitement des résidus.}

Certains projets miniers consistent à remanier des piles de déchets (souvent des résidus) de mines inactives ou abandonnées ou d'anciens tas de déchets dans les mines actives. Généralement, cela est proposé lorsque des méthodes plus efficaces d'enrichissement ont rendu économique l'extraction des métaux à partir des déchets miniers anciens. Le matériau des piles peut être envoyé pour traitement dans des installations sur le site même ou en dehors du site. Les projets miniers qui consistent uniquement à retraiter des piles de déchets de mines abandonnés, évitent les impacts environnementaux de l'exploitation à ciel ouvert et l'exploitation des placers, mais entraînent toujours des impacts environnementaux associés à la purification (enrichissement) des métaux dans les tas de déchets.

\subsection{4 Évacuation des morts terrains et des déchets de roche.}

Dans presque chaque projet, les minerais métalliques sont enfouis sous une couche de sol ordinaire ou de roches (appelée 'morts terrains' ou 'déchets de roche') qui doit être déplacée ou creusée pour permettre l'accès au dépôt de minerai métallique. Pour la plupart des projets miniers, la quantité de morts terrains générée par l'exploitation minière est énorme. Le rapport entre la quantité de morts terrains à la quantité de minerais (appelé 'taux de découverte') est généralement supérieur à un et peut être beaucoup plus élevé. Par exemple, si un projet minier proposé implique l'extraction de 100 millions de tonnes de minerai, le projet minier proposé pourrait générer plus d'un milliard de tonnes métriques de morts terrains et de déchets de roche.

Ces déchets volumineux, contenant parfois des niveaux significatifs de substances toxiques, sont généralement déposés sur place, soit en tas sur la surface ou comme remblai dans les carrières, ou dans les mines souterraines. Par conséquent, l'EIE d'un projet minier proposé doit évaluer soigneusement les options de gestion et les impacts associés à l'entreposage des morts-terrains.

\subsubsection{Extraction du minerai.}

Après qu'une compagnie minière a déplacé les morts terrains, l'extraction du minerai commence à l'aide d'équipements lourds et d'une machinerie spécialisés, tels que les chargeurs, les wagons de mine et les camions-benne, qui transportent le minerai vers les installations de traitement à travers des routes de transport des matériaux. Cette activité crée un groupe particulier d'impacts environnementaux, tels que les émissions de poussière fugitive des 
routes de transport des matériaux qu'une EIE pour un projet minier proposé devrait évaluer séparément.

\subsubsection{Enrichissement}

Bien que les minerais métalliques contiennent des niveaux élevés de métaux, ils produisent aussi de grandes quantités de déchets. Par exemple, la teneur en cuivre d'un minerai de cuivre de bonne qualité peut être seulement $0,25-0,50 \%$. La teneur en or d'un minerai d'or de bonne qualité peut être uniquement de quelques centièmes d'un pour cent. Par conséquent, l'étape suivante dans l'exploitation minière est le concassage (ou broyage) du minerai et la séparation des quantités relativement faibles de métaux du matériau non métallique du minerai au cours d'un processus de traitement dénommé enrichissement “. Le broyage est l'une des étapes les plus coûteuses de l'enrichissement et produit comme résultat de très fines particules qui facilitent une meilleure extraction du métal. Toutefois, le broyage permet également un dégagement plus complet des contaminants lorsque ces particules deviennent des résidus. Les résidus sont ce qui reste après le broyage du minerai en de fines particules suivi de l'extraction du métal/des métaux précieux.

L'Enrichissement inclut des techniques de séparation physique ou chimique comme la concentration par gravité, séparation magnétique, séparation électrostatique, flottation, extraction par solvant, extraction par voie électrolytique, lixiviation, précipitation et amalgamation (souvent impliquant l'utilisation du mercure). Les déchets provenant de ces processus incluent des décharges de déchets rocheux, des résidus, des matériaux de lessivage (pour les opérations d'or et d'argent) et des décharges de matériaux lessivés (pour les opérations de lessivage de cuivre).

La lixiviation impliquant l'utilisation de cyanure est un type de processus d'enrichissement, généralement utilisé pour des minerais d'or, d'argent et de cuivre et qui mérite une attention particulière en raison des graves impacts sur l'environnement et sur la sécurité publique. Avec la lixiviation, le minerai finement broyé est entassé dans de larges piles (dénommés 'piles de lixiviation') sur un coussin imperméable, et une solution contenant du cyanure est pulvérisé sur le sommet de la pile. La solution de cyanure dissous les métaux désirés et la liqueur mère contenant le métal est prélevée au bas de la pile à l'aide d'un système de tuyaux.

\subsection{7 Évacuation des résidus.}

Comme précédemment discuté, même les minerais métalliques à haute teneur sont composés presqu'entièrement de matériaux non métalliques et contiennent souvent des métaux toxiques indésirables (comme le cadmium, le plomb et l'arsenic). Le processus d'enrichissement génère de gros volumes de déchets appelé 'résidus,' c'est à dire les rejets d'un minerai après qu'il a été broyé et que les métaux désirés aient été extraits (par ex. avec le cyanure (or) ou l'acide sulfurique (cuivre). 
Si un projet minier implique l'extraction de quelques centaines de millions de tonnes métriques de minerai, le projet de mine générera une quantité similaire de résidus. La manière dont une compagnie minière dispose de son important volume de matériau de déchets toxique est l'une des questions centrales qui détermineront si un projet minier proposé est acceptable du point de vue environnemental. L'objectif à long terme primordial de la gestion et de l'entreposage des résidus est d'empêcher la mobilisation et le dégagement dans l'environnement des constituants toxiques des résidus miniers. Un sous-chapitre entier est consacré à une comparaison détaillée des options d'évacuation des résidus. Ces options incluent:

1. l'utilisation de bassins de décantation des résidus miniers ou 'bassin de réception des résidus.

2. l'assèchement et l'évacuation des résidus secs comme remblai.

3. l'entreposage sous-marin des résidus.

La première option (bassin de décantation des résidus) est de loin l'option la plus couramment utilisée, mais la deuxième option (élimination des résidus secs) est, dans la plupart des cas, l'option préférable du point de vue environnemental. La troisième option (entreposage sous-marin des résidus) est parfois proposée pour des mines situées près des environnements de mer profonde, ou dans de rares cas dans les lacs d'eau douce. L'entreposage sousmarin des résidus a eu un impact environnemental négatif dans les quelques cas où il a été pratiqué. Avant l'adoption de lois et normes environnementales, de nombreuses sociétés minières déversaient tout simplement les résidus dans l'emplacement le plus proche, y compris à proximité des rivières et des cours d'eau. Certaines des pires conséquences environnementales de l'exploitation minière ont été associées à la décharge à l'air libre des résidus, une pratique maintenant presqu'universellement rejetée. La société financière internationale (SFI) / la Banque mondiale explique:

"L'évacuation des résidus dans les cours d'eau (par ex., rivières, lacs et lagunes) ou en mer peu profonde n'est pas considérée comme une bonne pratique de l'industrie internationale. Par extension, le dragage des cours d'eau aux fins d'évacuation de déchets dans les lits fluviaux n'est également pas considéré comme une bonne pratique internationale." 4

Les Plans de réhabilitation et de fermeture des mines doivent décrire avec suffisamment de détails comment la compagnie minière restaurera le site d'une manière qui ressemble le plus possible à la condition environnementale d'avant exploitation; comment elle empêchera - à perpétuité - la libération des contaminants toxiques provenant de diverses installations minières (comme des puits à ciel ouvert abandonnés et des bassins de décantation des

4 IFC/World Bank (december 2007) "Environmental, Health and safety Guidelines for Mining."http:// www.ifc.org/ifcext/sustainability.nsf/AttachmentsByTitle/gui_EHsGuide lines2007_Mining/\$FILE/ Final+-+Mining.pdf. 
résidus miniers); et comment les fonds seront mis de côté pour s'assurer que les frais de réhabilitation et de fermeture seront payés à cet effet.

Un sous-chapitre entier de ce guide est consacré à une discussion sur la manière d'évaluer si oui ou non les plans de réhabilitation et de fermeture soumis par un promoteur de projet sont adéquats (Cfr section 3.7).

\section{TENTATIVE DE CLARIFICATION CONCEPTUELLE}

De l'époque coloniale à aujourd'hui, l'organisation politico-administrative de la RDC a subi des changements importants non seulement par rapport au découpage territorial, mais aussi et surtout en ce qui concerne les modes de gestion des entités locales (au moins quinze textes légaux).Deux principaux modes de gestion des entités publiques (centralisation, décentralisation) sont à élucider avant de voir en quoi consiste la décentralisation et saisir l'essence du développement endogène.

\section{a. Centralisation et décentralisation}

La centralisation est l'exacte opposée de la décentralisation, même si en pratique, la psychologie des acteurs et leur style de commandement peuvent créer des similitudes entre les deux concepts.

\section{b. La centralisation}

Dans un système administratif centralisé, toutes les décisions ont pour origine l'Etat, seule personne juridique reconnue et habilitée à les prendre sur toute l'étendue du territoire national. Toutes ces décisions administratives relèvent ainsi de la compétence des organes centraux, donc du pouvoir central. La centralisation consacre la présence des services publics dont la gestion est assurée par l'Etat lui-même. Elle entraîne, au niveau de la territoriale, la présence des fonctionnaires qui gèrent les affaires des entités locales au nom et pour le compte du pouvoir central, soumis au pouvoir hiérarchique de ce dernier et dont ils reçoivent instructions, ordres impératifs ou injonctions.

Un tel système administratif suppose que le pouvoir central peut adapter, du moins en théorie, son action à chaque partie du territoire national et à chaque unité de production. Dans la pratique cependant, cela n'est pas toujours le cas : la centralisation engendre des frustrations au niveau des entités locales ou des services publics de l'Etat, par la stérilisation ou le blocage de leurs initiatives. Ainsi donc, ce système est presque toujours atténué d'une déconcentration. La déconcentration se situe donc à mi-chemin entre la centralisation et la décentralisation. Dans un système administratif centralisé, elle est l'attribution aux entités locales (organes locaux) de certaines prérogatives du pouvoir central (organes centraux) sans que ces entités soient dotées d'une personnalité juridique distincte de celle de l'Etat. 
Actuellement, la décentralisation est l'un des points de divergence entre le pouvoir central et les provinces. ${ }^{5}$ En effet, la décentralisation en RDC se prépare dans un contexte de post-conflit où s'affrontent deux logiques, l'une unitariste et l'autre fédéraliste. La logique unitariste, favorable à une décentralisation administrative classique, est convaincue que la sauvegarde de l'unité nationale passe par l'existence, en province, des entités déconcentrées soumises au pouvoir hiérarchique de l'autorité centrale (district, territoire, cité, poste d'encadrement administration) ou de l'autorité provinciale (quartier, rue, groupement, village). La logique fédéraliste, qui prône la régionalisation, entend faire de la province une entité sui generis, pour ne pas dire politique.

Comme on peut s'en apercevoir, dans un système administratif où la déconcentration est de mise, le pouvoir de décision est accordé « à une autorité désignée et, comme telle, soumise à révocation (...) pour de simples motifs d'opportunité ». Bien plus, la déconcentration ne suppose, de la part du pouvoir central ou hiérarchique, un transfert des compétences et, à la fois, une capacité de coordination des activités sur l'ensemble du territoire. Souvent, il se crée des velléités de nature à provoquer la paralysie. C'est ainsi que la tendance générale est de remplacer la centralisation par la décentralisation.

La décentralisation est un procédé qui consiste à confier la gestion des services publics (décentralisation technique ou par service) ou des entités territoriales (décentralisation territoriale) à des organismes ou des organes dépendant du pouvoir créateur, mais jouissant visà-vis de ce pouvoir central de l'autonomie de gestion. La décentralisation émerge donc du souci de personnalisation ou d'individualisation des intérêts devant stimuler et rentabiliser la productivité de services publics ou des entités locales, en vue de l'amélioration des conditions d'existence de la population concernée. Elle repose ainsi l'idée d'une gestion par les administrés des affaires qui les concernent le plus directement.

En conséquence, la décentralisation devient un processus de responsabilisation qui nécessite beaucoup de volonté politique de la part du pouvoir central et de l'intelligence d'auto-actualisation en ce qui concerne les entités bénéficiaires.

La décentralisation territoriale implique que les entités territoriales décentralisées aient une personnalité juridique, des organes délibérants, un patrimoine propre et des autorités issues des élections. Son efficacité est fonction de la perception qu'on se fait mais aussi de la connaissance qu'on a de sa portée (pratique), pour éviter tout particularisme régional béat et toute atomisation inutile du pouvoir. ${ }^{6}$

\section{c. A propos du développement endogène des entités locales}

De nos jours, la littérature sur le développement a montré que la rationalité conceptuelle de ce terme est battue en brèche : les auteurs se rangent presque toujours dans un camp qui

5 Rapport général du Forum national sur la décentralisation, inédit, Kinshasa, octobre 2007:13.

6 Article 181 de la constitution du 18 février 2006. 
reflète le mieux leurs préoccupations souvent idéologiques. Depuis les années 1950, une vision économiste et/ou humanitariste affronte une autre qui se voulait alternative. ${ }^{7}$

Pour l'essentiel, nous pouvons dire que le développement pour nos entités locales dont la ville de Kolwezi, devrait être perçu comme un processus volontariste d'amélioration de la production des biens de consommation courante ayant pour but l'accroissement du pouvoir d'achat et des conditions de vie de chaque citoyen. Il s'agit des conditions adéquates d'existence humaine (habitation convenable, alimentation nutritive, hygiénique et équilibrée, installations sanitaires assainies, infrastructures de communication praticables, rhabillement commode, degré élevé de participation politique et de conscience du pouvoir,...). ${ }^{7}$ Cette appréhension pratique et dynamique du développement met en évidence le fait que le service territorial a l'obligation de s'occuper de tous les problèmes qui se posent au niveau des entités locales. ${ }^{8}$

En ce qui concerne la RDC, il est important de souligner le fait que l'incapacité de la bourgeoisie nationale de résoudre des problèmes vitaux de son environnement, pour des raisons multiples (extraversion des structures économiques, fraude fiscale, esprit de cueillette, corruption,...) met l'Etat et les entités locales décentralisées devant l'obligation de prendre en main le relèvement des conditions de vie de leurs citoyens.

En effet, la consolidation de la classe hégémonique reste aléatoire, son nombre relativement petit et concentré dans les villes, son assiette économique fragile et dépendante des structures du capitalisme périphérique; rongée par des contradictions, elle se complaît dans la consommation ostentatoire et accuse des carences dans la production effective des biens collectifs et des services publics. ${ }^{9}$

Cependant, les carences et l'impuissance des initiatives privées ne peuvent recommander l'omniprésence de l'Etat ou sa toute-puissance faisant recours à des méthodes d'action autoritaires et répressives comme sous la colonisation belge. Nous pensons néanmoins que l'administration territoriale, s'appuyant sur l'esprit de décentralisation et de bonne gouvernance, peut constituer cette armature dont le développement local a besoin. En effet, le développement est un phénomène polymorphe et concerne l'homme dans tous ses attributs, c'est-à-dire tout homme et tous les hommes pour la satisfaction de tous les besoins humains, économiques, politiques, culturels, sociaux et moraux.

7 Cahiers Zaïrois d'Etudes Politiques et Sociales n 4, mars 1984, pp. 33 et suivantes.voir aussi JeanPhilippe Peemans, Le développement des peuples face à la modernisation du monde, Paris/ Bruxelles, l'Harmattan/Academia Bruylaut.

8 Cette définition s'inspire de Banyaku Luape dans La problématique de l'intégration régionale en Afrique : Les objections sur la validité des théories courantes d'intégration régionale et les considérations empiriques sur la situation des Etats membres de la CPGL (Burundi-Rwanda-Zaïre), Thèse de doctorat en Relations Internationales, Lubumbashi, inédit, Université de Lubumbashi, 1982:111 et suivantes.

9 Les articles 3, 196, 201, 203 et 206 de la Constitution congolaise du 18 février 2006. 
A l'instar d'une plantule qui pousse des entrailles du sol local avant la photosynthèse, le développement économique et social souscrit au prescrit de l'endogénéité. Il est le produit d'un génie créateur autochtone et autonome.

Il s'agit donc d'un processus qui suppose, selon la perception de J.K. Galbraith : « action, c'est-à-dire mouvement vers un résultat ». " Une action pour le développement n'aura donc de chance de réussir que dans la mesure où la population y participe activement, dans la mesure où le développement est « saisi en main » par la population elle-même ». À cet égard, le développement des entités locales congolaises, dont la ville de Kolwezi, pour être endogène, suppose une action de masse pour la masse et doit être menée dans le cadre de la décentralisation dont il reçoit, en osmose, des effets bénéfiques. ${ }^{10}$ La décentralisation peut donc avoir des rapports étroits avec le développement endogène des entités locales.

\section{LA DECENTRALISATION ET LE DEVELOPPEMENT ENDOGENE DES ENTITES LOCALES}

Comme nous venons de le dire, le développement endogène des entités locales en RDC est tributaire du succès de la décentralisation. Phénomène polymorphe, global et globalisant, le développement s'identifie à chaque secteur de la vie sociale (secteurs politique, économique, culturel...).

Dans les lignes qui suivent, nous allons essayer de saisir l'impact de la décentralisation sur le développement politique, économique et culturel avant de jeter un regard sur sa structure en RDC.

\section{a. L'impact de la décentralisation sur le développement endogène}

Suivant l'accent qui est mis sur l'un ou l'autre aspect pratique de la décentralisation, on peut donc enregistrer un développement sectoriel donné. Il va sans dire que l'apprentissage de la décentralisation en constitue le moteur, et que, de son succès ou de son échec dépendent l'émancipation matérielle ou la pauvreté des entités locales congolaises. La décentralisation et le développement politique.

Le développement politique est un processus d'évolution qui vise une plus grande participation de la population à la vie politique, une meilleure intégration nationale et la prise de décision par des organismes gouvernementaux de plus en plus spécialisés et décentralisés.

Le choix des dirigeants par la population et de ses représentants (élection), la possibilité qu'a la population d'émettre des critiques constructives à l'encontre de ses dirigeants (liberté d'expression, liberté de presse, ...) constituent le reflet de la participation politique. Tandis que l'intégration nationale réfère à l'insertion des différentes entités locales aux circuits économiques et la communication spirituelle, c'est-à-dire la disparition des inégalités

10 Lobho Lwa Djugudjugu, «Le devenir de la pensée sociologique dans le Tiers-Monde », CIEDOP, Discussion Paper, nº 002188, Université de Kinshasa, 1988, pp. 14-17. 
criantes entre provinces, entre villes, entre communes et entre secteurs ou chefferies. ${ }^{11} \mathrm{La}$ prise de décision, elle, suppose le choix d'une alternative et l'application effective de celleci.

La « décentralisation-démocratie » nous met donc en présence des dirigeants ou technocrates qui connaissent mieux les réalités locales et qui peuvent mettre sur pied des programmes et projets réalistes et réalisables. Mais, depuis 1982 jusqu'à aujourd'hui, cela n'est pas encore une réalité dans les entités territoriales décentralisées : les hommes qui les animent ne sont pas toujours ceux que l'on attendait; la majorité d'entre eux ne s'adaptent pas encore à la décentralisation

A ce sujet, les discussions ont toujours été houleuses parmi les «ténors » de la décentralisation : faudrait-il placer dans les entités locales des originaires, des technocrates ou des opérateurs politiques? Sans chercher à engager une polémique là-dessus, nous pensons, pour notre part, qu'au nom du libre établissement garanti par la constitution (art. 30, al $1^{\mathrm{er}}$ ), le concept « d'originaire » est à redéfinir, et que le recours servile aux opérateurs politiques est à proscrire.

L'apprentissage de la décentralisation, pour son succès, devrait requérir la présence des hommes et des femmes qui en ont la vocation, les qualités, la technique et la psychologie; ce qui favoriserait des actions de nature à influer sur le développement économique et social de l'entité concernée.

\section{b. La décentralisation et le développement économique}

Dans le cadre de la présente étude, le développement économique est perçu comme un processus d'amélioration des conditions de vie des individus en leur fournissant ou en leur permettant d'obtenir les biens et services dont ils ont besoin.La décentralisation ouvre la voie aux programmes et projets locaux ou d'intérêt local. Au nom de la lutte contre les déséquilibres et inégalités régionaux, elle permet le désenclavement et la mise en place de nouvelles infrastructures (bâtiments administratifs, réseaux postaux et téléphoniques, essaimage des universités...). ${ }^{12}$

Il importe de souligner cependant que la décentralisation ne peut avoir un impact positif sur le développement économique que lorsque les acteurs opérationnels placés dans les entités provinciales et locales font usage d'imagination créatrice et de décision; car le développement économique implique action concrète, donc du travail. Il va sans dire que la création des conditions nécessaires à la formation de l'épargne locale (coopératives agricoles, d'éleveurs, de pêcheurs, d'épargne, notamment) devrait s'insérer dans le fil conducteur de tout processus de décentralisation visant le développement économique. Ce qui devrait inciter l'autorité locale à œuvrer pour la promotion de la petite et moyenne industrie

11 Lambinet, 1984 «projets de développement et administration locale au Zaïre », in Zaïre-Afrique, $\mathrm{n}^{\circ} 181$, op. cit., pp. 28-30.

12 Art. 181 de la Constitution de la République Démocratique du Congo. 
de transformation (huilerie, savonnerie, biscuiterie, menuiserie, cordonnerie, papeterie, ...), la production agricole et l'amélioration des voies de communication.

C'est à ce prix seulement que le «Congo profond » cessera d'être un mouroir pour devenir un véritable espace humanisé et socialisé susceptible d'attirer investisseurs et touristes.

\section{c. La décentralisation et le développement culturel.}

L'élargissement du cercle politique (démocratisation de la vie politique) et le rapprochement des centres d'impulsion de la population devraient, à coup sûr, transformer le mode de vie des citoyens des entités locales à la fois sur le plan matériel, intellectuel, moral et spirituel. Cette transformation des comportements globaux grâce aux « effets de contagion » de la décentralisation crée des besoins nouveaux. C'est ainsi, par exemple, que la population va de plus en plus s'intéresser aux questions relatives à l'emploi, à la médecine communautaire, à la création de nouvelles écoles (ou sections), à l'alphabétisation des adultes, à la construction ou réfection des routes, etc.

Comme on peut s'en apercevoir, le développement concerne tous les secteurs de la vie sociale, lesquels vivent en perpétuelle interaction. Le développement, atteint dans un secteur donné (par exemple le développement économique), influe nécessairement sur celui des autres secteurs (politique, culturel, notamment).

En ce qui concerne l'impact de la décentralisation sur le développement des entités locales, la délimitation que nous venons de proposer n'obéit qu'à une exigence didactique, théorique. Dans les faits, l'apprentissage de la décentralisation territoriale influe simultanément sur les différents secteurs de la vie sociale. La centralité de la territoriale et la transversalité de la décentralisation en constituent deux atouts majeurs.

A cet égard, la décentralisation territoriale peut être considérée à juste titre comme un véritable programme de développement. Mais en dépit de son adoption dans notre pays, les entités locales congolaises se meurent par manque des moyens financiers. Deux raisons essentielles expliquent cette situation. Il s'agit de la gloutonnerie du pouvoir central ou de l'échelon supérieur (par exemple : dans la ville de Kinshasa, l'Hôtel de ville prend l'essentiel des recettes au détriment des communes, pourtant décentralisées elles aussi). Et, d'une façon générale, il s'agit aussi de la mauvaise gouvernance économique et financière : l'orthodoxie ne semble pas avoir conquis les administrateurs. Des pratiques centralisatrices cohabitent encore avec la décentralisation territoriale.

A ces causes qui relèvent du comportement des acteurs opérationnels s'ajoutent des causes structurelles qui, elles, ont été à la base de l'échec de la décentralisation de 1982 en RDC. 


\section{d. Structure de la décentralisation en RDC}

Conformément à l'article 3 précité de la Constitution, outre la province, les entités territoriales suivantes sont décentralisées. Il s'agit de la ville, la commune et du secteur (ou chefferie). Cependant, la loi organique fixera leur composition, organisation et fonctionnement ainsi que leur rapport avec l'Etat (pouvoir de tutelle) et la province (pouvoir hiérarchique).

Au regard de la loi de 1982 et de ses modifications ultérieures, la RDC comprend 8374 entités territoriales dont 876 seulement (soit un peu plus de 10 pour cent) sont décentralisées comme l'indique le tableau ci-dessous.

La décentralisation territoriale se présente ainsi sous forme d'une structure pleine de cassures : la province est autonome c'est-à-dire décentralisée; sa subdivision, le district ne l'est pas; le territoire, subdivision du district ne sera plus décentralisé; le secteur (chefferie) est décentralisé pendant que les entités qui le composent, les groupements, ne le sont pas, encore moins les villages, leurs subdivisions. Au niveau des entités urbaines, la ville et ses subdivisions, les communes, sont décentralisées pendant que les quartiers et les rues ne le sont pas.

Lorsqu'on part verticalement de la province au village, on rencontre deux « bouchons centralisateurs », le district et le territoire. Le secteur, qui est décentralisé, se meut ainsi entre deux entités centralisées (le territoire et le groupement) où il existe des fonctionnaires dépendant du pouvoir central et/ou de la coutume. ${ }^{13}$

Ces entités (le district, le territoire et le groupement) risquaient, à la longue, de gêner la dynamique de la décentralisation, c'est-à-dire la reconstruction de la RDC à partir des entités locales. La Constitution vient d'élaguer le district en l'érigeant en province à moyen terme. Tandis que le territoire est ré-centralisé. Mais pour jouer quel rôle?

Au niveau de la ville, l'influence exagérée des animateurs des institutions urbaines (exécutif et législatif) peut avoir une incidence négative sur le fonctionnement des institutions communales. Est-il rationnel de décentraliser en même temps la ville et la commune tout en maintenant le quartier sous le régime de la centralisation?

La figure ci-dessous qui présente cette structure de la décentralisation montre clairement que la décentralisation n'est pas une réalité à la base.

La toute prochaine loi sur la décentralisation (voir Constitution, art.2 al.5 et art.3 al.4) devra redéfinir les relations de tutelle, hiérarchiques et fonctionnelles, en vidant des entités comme la ville, le territoire, le groupement et le quartier de tout venin d'immobilisme ou celui du gaspillage des ressources et de la fragmentation des initiatives.

L'amélioration des conditions de vie de l'ensemble de la population congolaise sur toute l'étendue du territoire national passe ainsi par un bon apprentissage de la décentralisation, c'est-à-dire (au-delà de l'autonomie de gestion des entités locales) « la dissémination à travers tout le pays des administrations, des industries ou organismes » qui se trouvent jusque-là concentrés à Kinshasa (la capitale) ou dans les provinces d'évacuation/d'entrée

13 Lotoy Ilango-Banga, 1995 «Le pouvoir traditionnel au Zaïre : Hier et aujourd'hui », in Bohambi, $\mathrm{n}^{\circ} 5$, mars, pp. 4-. 
des produits (Katanga et Bas-Congo). La segmentation de certaines entreprises publiques " sous forme de filiales autonomes à implanter dans chaque province " serait une bonne stratégie, à l'instar de la décentralisation de la recherche scientifique prônée par Jacques TSHIBWABWA. ${ }^{14}$ En effet, une bonne décentralisation s'accompagne toujours de la décentralisation des secteurs à disséminer dans les provinces, c'est-à-dire d'une certaine déconcentration.

Tout cela n'est pas encore une réalité. Ce qui fait que la décentralisation territoriale en RDC patauge encore et le développement de ses entités locales en pâtit. Il n'est plus nécessaire de rappeler que lorsque le processus de décentralisation tourne en rond (ou pas du tout), comme c'est le cas dans ce pays, au lieu du développement, c'est la stagnation, voire la régression, qui envahit ces entités locales. Pour ce faire, l'initiation à la mobilisation (économique) devrait requérir l'urgence.

\section{d. En quoi consiste la décentralisation territoriale?}

Comme nous l'avons dit, la décentralisation territoriale est un mode de gestion des entités locales qui consiste en une responsabilisation de celles-ci en ce qui concerne leur gestion quotidienne pour laquelle elles bénéficient d'une autonomie de décision.

De bonne heure, VUNDUAWE Te PEMAKO en avait dégagé les aspects pratiques pour saisir en quoi la décentralisation consistait sur les plans politique, économique et juridique.

Sur le plan politique, la décentralisation est le corollaire de la démocratie : elle favorise la formation du citoyen (électeur ou élu) par le jeu et les enjeux d'une élection, et lui permet de maîtriser les rouages du pouvoir et les astuces de la technique (la psychologie aussi) par le jeu d'essai-erreur au niveau le plus bas de la gestion des affaires publiques. La décentralisation territoriale est ainsi perçue comme une véritable école politique. Elle est en effet un facteur d'épanouissement de l'esprit civique et peut permettre, dans le chef des citoyens, l'émergence d'une conscience du pouvoir. Sur le plan économique, décentraliser, c'est assurer un développement harmonieux et équilibré des provinces et autres entités territoriales décentralisées.

Le droit de prendre l'initiative en matière de programmes et projets de développement économique, accordée aux entités locales, ouvre la voie au privilège des programmes ou projets locaux, donc à un développement national équilibré. ${ }^{5}$ La décentralisation territoriale contribue ainsi à la promotion de la participation locale qui, elle, permet la mobilisation et la valorisation des ressources locales ainsi qu'une meilleure coordination des activités de développement. Sur le plan juridique ou administratif, décentraliser, c'est transformer en

14 Omombo Omana, 2007, « Comment financer la décentralisation? », in La Référence Plus, $\mathrm{n}^{\circ} 4072$ du 6 septembre, p.2. Tshibwabwa Kuditshini, J., « Mondialisation et développement démocratique : Vers la relégitimation du rôle du secteur public en Afrique », in Conférence Guy Mhone sur «Les réformes du secteur public en Afrique : Rétrospective et perspectives », Document de travail, Zomba, CODESRIA. 
centres d'impulsion les entités locales (provinces, villes, communes et secteurs) qui cessent d'être de simples circonscriptions politico-administratives pour devenir des personnes morales de droit public, avec tous les attributs de la personnalité juridique : ester en justice, exercer des prérogatives de puissance publique, prendre des décisions adaptées au besoin des administrés ou aux circonstances.

A ces trois aspects pratiques ou manifestations concrètes de la décentralisation, on peut ajouter un quatrième qui résulterait de sa pratique-même et revêtirait une coloration opérationnelle, managériale pour ainsi dire. Sous cet angle, décentraliser, c'est motiver. « En effet, le degré de responsabilisation d'un individu et la liberté d'action qui lui est laissée peuvent être à la base d'une prise de conscience capable de stimuler sa productivité ». Cela est vrai aussi pour une entité rendue autonome par le moyen de la décentralisation. Celle-ci constitue en effet un processus d'auto-identification des autorités locales élues aux objectifs de reconstruction au niveau local, motivées ainsi par le souci de leur réélection.

L'analyse des aspects pratiques de la décentralisation territoriale a pour mérite de relever le fait que, lorsque le recrutement (nomination ou élection) au sein du service territorial est réalisé en tenant compte du caractère incitateur de la responsabilisation des entités locales, l'administration territoriale va demeurer l'épine dorsale du développement national ou local dont il convient d'appréhender le contenu.

\section{L'IMPERATIF DE LA MOBILISATION DES MASSES LABORIEUSES}

Comme nous l'avons dit précédemment, le développement endogène implique une action de masse pour la masse. Il est donc question d'amener la population à pourvoir, par sa propre action, à ses besoins essentiels tout en contribuant au développement de sa communauté. Il s'agit en fait pour l'administration territoriale d'inciter la population à participer non seulement aux activités politiques (par exemple : les élections) mais aussi à la réalisation des programmes ou projets locaux arrêtés.

En effet, la mobilisation de masses laborieuses demeure, à bien d'égards, « un excellent moyen d'aborder les problèmes de développement économique et social, étant donné que la solidarité internationale est affublée d'objectifs économiques et expansionnistes $\gg{ }^{15}$ Par conséquent, seul l'effort scientifique, inventif et productif des congolais en priorité peut permettre de panser les plaies du pillage et de reconstruire les infrastructures détruites par la belligérance.

La mobilisation populaire s'impose ainsi comme une stratégie pouvant stimuler la croissance à partir des entités locales, tout en améliorant la qualité de vie dans le milieu rural. Cependant, la nature des objectifs de l'Etat, ou parfois ceux de l'autorité de tutelle (pouvoir central), peut anéantir ou pervertir les objectifs et l'efficacité de la mobilisation. Le cursus historique de la RDC laisse entrevoir le fait que l'efficacité de la mobilisation des

15 Lotoy Ilango Banga et Ingange Ikyo, « Contribution des chefs coutumiers au développement socio-économique de l'hinterland Tumba-maindombe », in Mouvements et Enjeux Sociaux, $\mathrm{n}^{\circ} 18$, juillet-août. 
masses laborieuses (entant que stratégie d'auto-prise en charge) et sa nature sont intimement liées à la nature du pouvoir d'Etat.

\section{a. La mobilisation des masses laborieuses à l'époque coloniale}

D’une façon générale, la colonisation devait se financer par des ressources générées par les colonies. En ce qui concerne la colonisation belge, le principe de l'union personnelle (Etat Indépendant du Congo : 1885-1908) et celui de l'autonomie budgétaire consacré par la charte coloniale (Congo-Belge:1908-1960) ont constitué le fondement juridique du recours au potentiel productif de la population. Tous les Hommes Adultes Valides (HAV) étaient ainsi astreints à des prestations annuelles individuelles et au paiement de l'impôt en numéraire.

Le système de prélèvement sur l'effort productif des populations (rurales) était tellement efficace que les investissements publics fussent presque entièrement financés par le moyen du recours au potentiel productif de la population et aux ressources de la colonie.

Ainsi donc, la récolte du caoutchouc rouge et du copal fossile, les cultures obligatoires et l'effort de guerre (notamment) ont permis à la Belgique de faire face à la grande crise économique des années 1929-1930 et de sortir de la Deuxième Guerre Mondiale presque sans dette. ${ }^{16}$ Le secret de ce succès est sans nul doute lié aux objectifs d'une colonie d'exploitation ouverte aux investissements directs étrangers bien sûrs mais surtout soutenue par un système administratif de proximité et travaillant suivant la logique d'une économie extravertie. Ce qui explique le fait que l'intensification de la production se soit toujours organisée au détriment des producteurs.

Pendant la colonisation belge, le développement économique du Congo a été essentiellement l'œuvre des masses laborieuses autochtones grâce à la mobilisation de leur potentiel productif pour des fins étatiques. ${ }^{17}$ Que va-t-il se passer après la colonisation belge?

\section{a. La mobilisation des masses laborieuses après l'indépendance}

Le recours au potentiel productif de la population pendant la colonisation n'a pu améliorer les conditions de vie des masses congolaises. Vers les années 1945, le colonisateur belge tenta en vain d'y remédier par la création du fonds du bien-être indigène.

Cependant après l'indépendance, l'engouement des Congolais vers les activités politiques n'a pas permis une actualisation conséquente du recours au potentiel productif de la population. C'est ainsi qu'au lendemain du 30 juin 1960, l'indépendance ayant pris une

16 Leclercq, H., 965, « Un mode de mobilisation des ressources : le système fiscal. Le cas du Congo pendant la période coloniale », in Cahiers Economiques et Sociaux, Vol. III, $\mathrm{n}^{\circ}$ 2, juin 1, pp. 106-108.

17 Décret du 18 novembre 1903, décret de 1908 sur l'impôt en numéraire, décret du 20 février 1917 sur les cultures obligatoires, décret du 5 décembre 1933 sur le travail forcé qui a été renforcé en 1942 pour l'effort de guerre. 
connotation péjorative, l'idée de recourir à l'effort productif des autochtones était tout simplement contraire à la notion de « liberté », comprise par les congolais comme leur libération de « toute forme d'exploitation ». D'ailleurs, une aire populaire exprime mieux cette contradiction lorsqu'on chante en chœur : « Nous voulons être pauvres dans la liberté. Nous refusons d'être riches dans la servitude », tout en oubliant qu'en réalité, il n'y a point de liberté dans la pauvreté.

Comme on peut le remarquer, dès le 30 juin 1960, le jour de son accession à sa souveraineté, le Congo tourna le dos au travail productif. La mobilisation populaire s'organisa ainsi autour des objectifs essentiellement politiques (élection, rébellion, délation). La nécessité de consolidation du pouvoir qui s'observera à partir de 1966 déclencha le processus de sa personnalisation et de sa concentration entre les mains d'une oligarchie friande de prestiges et d'honneurs.

C'est ainsi que malgré les vœux pieux qui appelaient les Congolais de « retourner à la terre », la mobilisation sous l'impulsion du monolithisme revêtira son auréole politique. Les Congolais seront donc conviés à chanter et à danser (à peu près comme la cigale) pour la gloire du guide éclairé et de ses acolytes. Ainsi donc, l'animation politique va se hisser au rang d'une technologie à exporter et supplanter l'animation agricole.

Pendant plus de quatre décennies, les Congolais en sont encore là. Plongés dans l'attentisme, ils continuent encore à prendre abusivement potentialités pour richesses et à se méfier du travail productif au profit du courtage et de l'évangile de prospérité. Le pays tend à tourner en rond et l'histoire semble se répéter. Ce qui rend toujours actuelle la pertinence de ce constat de Elikia Mbokolo : Au Zaïre, la crise politique et économique commença dès l'indépendance et ne devait connaître que de brefs répits ... De 1960 à 1965, elle fut dominée par la lutte entre les deux tendances radicale et modérée du mouvement national pour s'assurer le contrôle exclusif du pouvoir : sur cette contradiction majeure, sont venus se greffer, pour en aggraver les effets, de nombreux antagonismes tels que les clivages ethniques, la compétition entre les grandes puissances, les désaccords au sein même de chacune des tendances. ${ }^{18}$

Il va sans dire que la transition dite démocratique, avec des alliances qui se faisaient et se défaisaient, est restée pour l'histoire une réplique incontestable des années 1960. Le souci du contrôle exclusif du pouvoir a toujours fait oublier à la classe politique congolaise la misère du peuple en refusant à l'inciter à une auto-actualisation en vue de son mieux-être. D'ailleurs, l'une des causes de l'échec des programmes et projets de développement initiés par les instances gouvernementales reste le manque d'adhésion de la population. Le lien entre les gouvernants et les gouvernés étant faible, la participation de ceux-ci demeure timide. $^{22}$

18 Lofembe Benkenya, 2006, « Les exiges du développement de la RDC. Essai de sociologie du développement », in Les sciences sociales face à la lutte contre la pauvreté en RDC, Actes des troisièmes journées scientifiques de la Faculté des Sciences sociales de l'université de Kinshasa, Kinshasa, inédit, pp.123-134. 
Les choses étant telles que nous venons de le voir, que faire pour amener l'homme congolais à s'auto-actualiser, à puiser dans son environnement et par sa propre action ce dont il a besoin, c'est-à-dire à contribuer au développement national et à même temps à améliorer ses conditions d'existence

b. La mobilisation des masses laborieuses comme stratégie de développement endogène

Sous son aspect technique, la mobilisation des masses laborieuses est une stratégie efficace pouvant permettre l'augmentation de la production et l'amélioration des conditions d'existence humaine. Elle donne ainsi un pouvoir d'achat aux populations concernées.

A l'époque coloniale, à cause de son insertion dans le système colonial, la mobilisation de l'effort productif de la population s'était organisée conformément à la logique prédatrice. ${ }^{19}$

Par contre, après l'indépendance, les objectifs économiques seront étouffés au profit des objectifs politiques. D'où l'importance accordée à la mobilisation politique et la précarité qui cohabite avec la population.

A ce stade, nous pouvons nous demander s'il est possible aujourd'hui de s'inspirer de la technique de mobilisation coloniale en vue d'une relance endogène de l'économie congolaise. Comment peut-on y parvenir?

Deux conditions essentielles peuvent garantir la capitalisation de la mobilisation coloniale. Il s'agit de la connaissance des techniques d'application de cette mobilisation et d'une réelle volonté politique qui mettent l'économie nationale au service du congolais. Désormais, il ne s'agira plus d'utiliser des méthodes autoritaires de l'époque coloniale mais des méthodes persuasives et incitatives, pour amener la population à contribuer massivement et positivement à l'effort de développement national ou local.

L'administration locale devra donc concentrer ses efforts sur l'animation agricole, sanitaire et coopérative, pour asseoir une éducation de masse appropriée. En tant que partenaires privilégiés du développement endogène, les partis politiques ont une grande part de responsabilité à ce niveau. En se sentant concernés par cette nouvelle vision de l'administration territoriale, ils devront initier ou former leurs cadres à ce type de gestion pour ne proposer aux suffrages populaires que les meilleurs d'entre eux. Aussi, le recrutement au sein du service territorial devrait-il tenir compte des critères de compétence et d'esprit d'initiatives, et prévoir la formation permanente du personnel tout au long de la carrière. Ce qui pourrait donner à ce personnel des aptitudes à la coordination ou à la collaboration, à l'analyse des besoins et des ressources du milieu, à la conception et à la gestion des projets locaux de développement et à inciter la population à y participer massivement ainsi qu'à pourvoir, par sa propre action, à ses besoins essentiels (Bulu Bobina 1984:27-28).

19 J. Kankwenda Mbaya, 2005, L'économie politique de la prédation au Congo Kinshasa. Des origines à nos jours (1885-2003), Kinshasa-Monréal-Washington, ICREDES, pp. 8, 19-36. 
Dans les entités locales congolaises, la mobilisation devrait s'entendre comme la mise au travail des forces productives (paysans adultes valides) en vue d'une plus grande productivité ayant pour effet la réalisation d'objectifs d'intérêt commun et l'amélioration de leurs conditions d'existence.

Sous la colonisation belge, le recours au potentiel productif de la population ou la mobilisation des masses laborieuses avait permis au colonisateur de maîtriser l'instabilité économique des années 1929-1930, de faire face aux « dégâts économiques » provoqués par la Deuxième Guerre Mondiale (effort de guerre) et de hisser le Congo-Belge au rang de puissance économique en Afrique noire. La mobilisation économique, pour servir d'outil au programme de décentralisation en vue de l'émancipation matérielle des entités locales congolaises, doit être une préoccupation permanente, une opération continue et avoir des objectifs globaux précis. Car la participation active et effective de la population aux programmes et projets de développement arrêtés est une condition essentielle pour le succès de ceux-ci. C'est cette participation en effet qui peut donner au développement atteint ou à atteindre son caractère endogène.

\section{CONCLUSION}

L'option levée par le constituant congolais en faveur de la décentralisation renvoie à l'idée du choix politique opéré en faveur d'une stratégie de développement de la RDC à partir des entités locales. Cela suppose une prise de conscience effective dans le chef du pouvoir central qui devrait se départir de tout esprit centralisateur ou de complaisance. En effet, « les solutions aux problèmes ne peuvent être commandées (centralisation). Elles doivent être découvertes, trouvées sur base de l'imagination, de l'expérimentation et de la critique, sur base de la décentralisation $» .^{20}$ Pour que le développement de la RDC se réalise à partir des entités territoriales décentralisées, il faudra que des préoccupations de production économique et de bien-être social soient intériorisées par les pouvoirs publics au niveau central et au niveau local. La reconversion des mentalités, à tous ces niveaux, devrait demeurer le cheval de bataille des acteurs concernés dans le processus de décentralisation. Sinon, au lieu d'intensifier la production économique, la décentralisation et l'autonomie des entités locales vont intensifier des conflits de tout genre et la stagnation.

Mettre l'économie au service du peuple en laissant l'autonomie aux initiatives des entités territoriales décentralisées, malgré le rayonnement et le prestige que cela peut susciter en faveur des autorités provinciales ou locales, serait une preuve éloquente d'une volonté politique manifeste de la part du pouvoir central. Le Congo post-conflit en a besoin pour sa reconstruction effective et durable. Dans cette perspective de globalisation, beaucoup d'initiatives voient le jour pour exiger des entreprises internationales des procédures, une certaine ligne de conduite et leurs propres responsabilités sociales à respecter, partout où elles opèrent. Ainsi donc, l'initiative de transparence des industries extractives (ITIE) préconise 
l'intérêt des entreprises à divulguer les paiements qu'elles versent aux gouvernements et d'agir dans la transparence lors d'accords contractuels. Nous sommes en présence d'une question de s'autodéterminer autrement, en appelant les uns et les autres à plus de justice et d'éthique dans les affaires Il faut noter pour ne point oublier que pour toute exploitation minière, l'Etude d'impact environnemental et le plan de gestion environnementale du projet, doivent être déposés en même temps que la demande du droit d'exploitation. Leur approbation par l'autorité compétente, est une condition d'octroi du droit d'exploitation. La R.D.C., scandale géologique mondial présente des impacts sociaux dans ses zones minières, impacts qui retiennent l'attention de l'opinion tant nationale qu'internationale. Les zones de la RDC étaient sans nul doute des îlots de développés. En effet, le taux de scolarité, d'accès à l'eau et à l'énergie électrique ainsi qu'aux soins de santé, était de loin supérieur au reste du pays.

\section{BIBLIOGRAPHIE}

1. Banyaku, L. et al., 2004, Prospectus électoral : estimations démographiques et documentation sur les modes de scrutin, tome 2, Kinshasa, PUK.

2. Banyaku, L., 1984, « Le développement économique : sa rationalité conceptuelle et les modèles théoriques du processus cumulatif et progressif », in Cahiers Zaïrois d'Etudes Politiques et Sociales $\mathrm{n}^{\circ} 4$, mars.

3. Banyaku, L., 1982, La problématique de l'intégration régionale en Afrique : les objections sur la validité des théories courantes d'intégration régionale et les considérations empiriques sur la situation des Etats membres de la CPGL (Burundi-Rwanda-Zaïre), Thèse de doctorat en Relations Internationales, Lubumbashi, inédit, Université de Lubumbashi.

4. Birangamoya, Makombe, 1984, «Décentralisation et développement au Zaïre : la répartition des compétences en matière de développement », in Zä̈re-Afrique, $\mathrm{n}^{\circ} 181$.

5. Bulu-Bobina, B., 1984, "Formation et décentralisation territoriale : regard sur un aspect du développement rural au Zaïre », in Zä̈re-Afrique, $\mathrm{n}^{\circ} 187$, septembre.

6. De Saint Moulin, L., 1988, « Histoire de l'organisation administrative du Zaïre », in Zaïre-Afrique, $\mathrm{n}^{\circ} 224$, avril.

7. Ekili, T., et Otemikongo M., 1990, « La décentralisation administrative et les finances publiques zaïroises (cas de la région du Haut-Zaïre », in Zaïre-Afrique, $\mathrm{n}^{\circ}$ 242, février.

8. Mbokolo, E., 1980, L'Afrique au XXe siècle : le continent convoité, Paris-Montréal, Etude vivante.

9. Epee, G., et Otemikongo M., 1992, « Entités territoriales décentralisées et financement public du développement local au Zaïre », in Zaïre-Afrique, $\mathrm{n}^{\circ} 266$, juin-juillet-août.

10. Galbraith, J. K., 1964, Les conditions du développement économique, Paris, Nouveaux Horizons.

11. Ilunga, K., 1980, « Interdisciplinarité et politique : pour une science politique africaine du temps présent », in Cahiers Economiques et Sociaux, vol. XVIII, n 2, juin. 
12. Isango, I.W., 1991, " Décentralisation territoriale et pratiques centralisatrices au Zaïre », in Zaïre-Afrique, $\mathrm{n}^{\circ}$ 258, octobre, pp. 421-435.

13. Isango, I.W., 1988, « La décentralisation administrative pour le développement : quelques écueils à éviter », in Zaïre-Afrique, ${ }^{\circ} 222$, février, pp. 85- ISANGO

14. Kankwenda, M., 2005, L'économie politique de la prédation au Congo Kinshasa. Des origines à nos jours (1885-2003), Kinshasa-Montréal-Washintongton, ICREDES.

15. Kanyinda, L., « La décentralisation territoriale zaïroise à l'épreuve de la théorie et des faits », in Les Cahiers du CEDAF, n 2, avril 1984.

16. Lambinet, F., 1984, « Projets de développement et administration locale au Zaïre », in Zaïre-Afrique, $\mathrm{n}^{\circ} 181$.

17. Leclercq, H., 1965, «Un mode de mobilisation des ressources : le système fiscal. Le cas du Congo pendant la période coloniale », in Cahiers Economiques et Sociaux, Vol. III, $n^{\circ} 2$, juin.

18. Lobho, L.D., 1988, " Le devenir de la pensée sociologique dans le Tiers-Monde », CIEDOP, Discussion Paper, $n^{\circ}$ 002/88, Université de Kinshasa.

19. Lofembe, B., 2006, « Les exigences du développement de la RDC. Essai de sociologie du développement ", in Les sciences sociales face à la lutte contre la pauvreté en $R D C$, Actes des troisièmes journées scientifiques de la Faculté des Sciences Sociales de l'Université de Kinshasa, Kinshasa, inédit.

20. Lotoy, I.-B. et Ingange, I., 2004, « Contribution des chefs coutumiers au développement socio-économique de l'hinterland Tumba-maindombe ", in Mouvements et Enjeux Sociaux, n 18, juillet-août.

21. Lotoy, I.-B. et Mukika, E., 1989, « Pour un nouveau type d'homme adapté à la décentralisation. Contribution à l'analyse de l'efficacité de la territoriale au Zaïre », in ZaïreAfrique, $\mathrm{n}^{\circ} 239$, novembre.

22. Lotoy, I.-B., 1995, « Le pouvoir traditionnel au Zaïre : Hier et aujourd'hui », in Bohambi, $\mathrm{n}^{\circ} 5$.

23. Mulumbati, N., 2006, Introduction à la science politique, $2^{\mathrm{e}}$ édition, Lubumbashi, Ed. Africa.

24. Mulumbati, N., 1980, Manuel de sociologie générale, Lubumbashi, Ed. Africa.

25. Muyer, O., 1986, Impératif du développement et réforme de l'administration locale au Zaïre, Kinshasa, PUZ.

26. Omombo, O., 2007, « Comment financer la décentralisation? », in La Référence Plus, $\mathrm{n}^{\circ} 4072 \mathrm{du} 6$ septembre.

27. Peemans, J.-P., 2002, Le développement des peuples face à la modernisation du monde, Paris/Bruxelles, l'Harmattan/Academia Bruylaut.

28. Tshibwabwa K., 2007, « Mondialisation et développement démocratique : Vers la relégitimation du rôle du secteur public en Afrique », in Conférence Guy Mhone sur « Les réformes du secteur public en Afrique : Rétrospective et perspectives », Document de travail, Zomba, CODESRIA.

29. Vie, J.-E., 1986, Les sept plaies de la décentralisation, Paris, Economica. 
30. Vunduawe, T.P., 1982, « La décentralisation territoriale des responsabilités au Zaïre. Pourquoi et comment? II. La nouvelle organisation territoriale, politique et administrative du Zaïre : ses motivations et sa portée exacte ", in ZaïreAfrique, $n^{\circ} 166$, juinjuillet-août.

31. Yamba Yamba, S., 2005, Les dynamiques de la décentralisation en RDC, Kinshasa, inédit. 